\title{
El outsourcing de los sistemas de información en las organizaciones públicas
}

\author{
Andrade Castro, Jesús Alberto*- \\ Mandrillo, Cósimo** \\ * Economista. Magister en Computación Aplicada y Master in Management Information \\ Systems. Profesor Titular y Director del Departamento de Computación de la Facultad \\ Experimental de Ciencias. Universidad del Zulia (LUZ). Maracaibo, Venezuela. Acreditado \\ en el Programa de Promoción al Investigador (PPI) del FONACIT. E-mail: \\ jandrade@luz.edu.ve, jandrade@luz.ve, jandrade01@yahoo.com \\ ** Licenciado en Letras. Magíster en Literatura Latinoamericana. Doctor en Literatura \\ Hispanoamericana. Experto en hipertextualidad. Profesor Titular (Emérito) de la Facultad \\ de Humanidades y Educación (LUZ). Acreditado en el PPI. E-mail: cosimom@cantv.net
}

\section{Resumen}

El desarrollo de los sistemas de información (SI) siempre ha involucrado a múltiples personas con diversas formaciones e intereses, sin embargo, los trabajos acerca de los SI se han centrado en dos grupos: los técnicos que desarrollan aplicaciones y sistemas, de una parte y, los usuarios de la otra. Las organizaciones aplican políticas estratégicas para procesar la información, una de ellas es el outsourcing la cual es utilizada como herramienta para desarrollar productos, aplicaciones de software y SI, todo dentro de una práctica organizacional que es moldeada por las tecnologías y los sistemas de información (TSI). De esa manera, se establecen relaciones de poder y control que son incrementadas por el conocimiento técnico que se posee y que se expresa en los hábitos que en forma rutinaria desarrollan las personas en su práctica organizacional. Este trabajo hace un análisis teórico interpretativo sustentado en la Teoría de la Estructuración de Anthony Giddens que considerar los valores, creencias y construcción social de significados. Por ello, esta investigación es una contribución teórica que se basa en la interpretación de las prácticas organizacionales cuando se ejecutan políticas de outsourcing en el desarrollo de sistemas de información en la administración pública. Las reflexiones finales que se alcanzan, muestran que las personas con conocimiento técnico utilizan las prácticas organizacionales para acentuar sus intereses y afianzar su poder.

Palabras clave: Sistemas de información, poder, práctica organizacional, teoría de la estructuración, outsourcing. 


\title{
Outsourcing of I nformation Systems in Public Organizations
}

\begin{abstract}
The development of information systems (IS) has always involved multiple people with diverse formations and interests; however, works about IS have focused on two groups: on the one hand, technical works, devoted to the development of applications and systems, and on the other hand, users. Organizations apply strategic policies to process information; one of them is outsourcing, which is used as a tool to create knowledge, to develop products, software applications and IS, all done within an organizational practice modeled by technologies and information systems (TIS). Thus, power and control relations are established, which are increased by technical knowledge possessed by people; these are expressed in habits that, routinely, people develop in their organizational practice. The approach that is used contributes to the increase of our understanding of the IS in terms of the relationships among organizations. This research is supported by Anthony Giddens' Structuration Theory, as well as by the use of interpretative methodology that consider value, beliefs and the social construction of meanings. For this reason, this thesis is essentially a methodological contribution to the interpretation of social construction through organizational practices when information systems are developed.
\end{abstract}

Key words: Information systems, power, organizational practice, structuration theory, outsourcing.

Recibido: 04-07-08. Aceptado: 04-11-16

\section{I ntroducción}

Esta trabajo aborda el tema de los sistemas de información (SI) desde una perspectiva social. En particular, analizamos las relaciones de poder en las organizaciones que aplican políticas de alianzas para desarrollar sistemas de información. Eso significa que investigamos los sistemas y tecnologías de información en contradicción con la gran mayoría de los estudios que se concentran en el desarrollo técnico de aplicaciones y el análisis del impacto que éstas producen en las organizaciones. Esta investigación intenta demostrar que los sistemas y tecnologías de información (TSI) se institucionalizan a través del poder, lo cual se explica al examinar la dimensión política de las organizaciones y los elementos que la conforman. 
Para Giddens (1989), el poder se ejerce sobre otros, pero no como una relación entre individuos, sino como una cualidad de la vida institucional, que es reproducida crónicamente por las prácticas comunicativas del día a día que ejercen los miembros de las instituciones. La definición de poder que aquí usamos está asociada a la posibilidad política de tomar decisiones y no como el mero ejercicio de controlar a los demás. Evidentemente, no pretendemos negar la existencia de un poder que se ejerce en función del control manifiesto en las relaciones entre los actores sociales de la organización, sino que en este trabajo le damos relevancia, a la posibilidad que tienen los actores de decidir acerca de las políticas organizacionales. Así que, el análisis se sustenta no en el control individual que un sujeto tiene sobre otro, sino en la potencialidad que tienen algunos individuos para decidir el destino de la organización. Es así como, el poder es determinante en la gestión de los sistemas de información, porque los sistemas forman parte de los elementos ideológicos que se institucionalizan en la organización.

En el contexto de la gestión de sistemas de información en las organizaciones existen dos tendencias para considerar los problemas, una en forma lineal y determinista (relación causa efecto) y otra que es socialmente construida (interpretativa). Con este trabajo buscamos una explicación política al fenómeno de las tecnologías de información y la acción humana en el contexto de las organizaciones, alejada de los modelos deterministas, que tratan de comprobar qué agentes causales producen algún tipo de impacto en la organización, por lo tanto se trata de un análisis interpretativo de la realidad.

\section{Investigación de los sistemas de información y la Teoría de la Estructuración}

El estudio de los sistemas de información (SI) corresponde a un campo aplicado orientado al desarrollo de sistemas y aplicaciones en organizaciones que usan tecnologías de información. Por consiguiente, el principal laboratorio para indagar acerca de los SI es la organización.

La frontera existente entre organizaciones y tecnologías de información es muy difusa y difícil de separar. Quizás por eso, se suelen publicar artículos acerca de tecnologías y sistemas de información en revistas especializadas en temas organizacionales. Lo mismo ocurre con temas acerca de organizaciones que son publicados en revistas de tecnologías y sistemas de información. Los estudios acerca de las organizaciones, sin embargo, han tenido mayor influencia sobre los temas de sistemas y tecnologías de información que al revés. Este desbalance de influencia es razonable porque la investigación en tecnologías de información se centra en los SI en organizaciones, en tratar de entender cómo los 
fenómenos organizacionales afectan el desarrollo y uso de tecnologías y cómo los sistemas tecnológicos de información moldean a las organizaciones. Por el contrario, el tema central de los investigadores de las organizaciones no son las tecnologías y sistemas de información, sino los tópicos relacionados con la toma de decisiones, liderazgo, dinámicas de grupos, estructuras organizacionales, entre otros. Con todo lo anterior, los temas asociados a ambas disciplinas tienden a solaparse en lugar de separarse y las diferencias existentes entre ambos campos son más de naturaleza epistemológica.

Tradicionalmente, la disciplina de los sistemas de información ha concentrado sus esfuerzos en modelos de organización, métodos y herramientas para el desarrollo de aplicaciones técnicamente efectivas y eficientes. En ese sentido, los SI han madurado en función de tres disciplinas que están asociadas: ciencias de la computación, ciencias gerenciales y teoría organizacional. El estudio de los sistemas de información como disciplina tiene su origen en la incapacidad manifiesta de las ciencias de la computación para resolver problemas de aplicación en organizaciones. Mientras las ciencias de la computación se centran en la exploración y estudio de la tecnología digital en base a programas lógicos, los SI se centran en el uso de computadores para resolver problemas asociados a procedimientos organizacionales y toma de decisiones.

El estudio científico de los factores asociados a las tecnologías, sistemas y organizaciones intenta separar -por lo general- los aspectos técnicos de los aspectos sociales, como si se tratara de elementos claramente separables. Y como la eficiencia tecnológica muchas veces es contrastada con la ineficiencia de las organizaciones, con frecuencia, se intenta buscar soluciones técnicas a problemas de índole social o política, o buscar soluciones políticas y sociales a problemas de estricto orden técnico. Por ello, para ese tipo de situaciones se suele encontrar una línea que diferencia el mundo social del técnico.

La disciplina de los sistemas de información es conducida básicamente por los elementos tecnológicos y ello por la necesidad de producir y diseñar buenas soluciones prácticas (aplicaciones) asociadas a las tecnologías de información. Los SI sirven, entonces, para legitimar prácticas organizacionales a través del uso de la tecnología al convertir procesos difíciles y complejos en tareas sencillas y rutinarias. “Tecnología y organización tienen, entonces, algo en común: convertir lo complejo en simple, lo inusual en rutinario, lo distante en algo que está a la mano, disponible" (Malavé, 1999:55). Analizar procesos de las organizaciones implica describir secuencias de acciones asociadas a las tecnologías usadas, y eso significa reconocer que tecnología y organización tienen en común la manera de representar y transformar la realidad a través de la práctica organizacional. 
La teoría social, en general, tiene un rol importante en el desarrollo de los sistemas de información. En la tradición estructuralista el énfasis está en las estructuras, mientras que en la tradición fenomenológica y hermenéutica, los seres (agentes) humanos son el centro de atención. Lo ideal es encontrar una teoría que permita el acercamiento entre ambas posturas, donde el análisis de lo social esté relacionado inseparablemente con las estructuras organizacionales y la relación recurrente entre el flujo constante de acciones y el conjunto de tradiciones institucionalizadas, resulte tan estrecha que moldee la práctica humana, a la vez que refuerce las estructuras institucionales.

La Teoría de la Estructuración propuesta por Giddens (1989) ofrece una alternativa al fenómeno social, pues incorpora la interpretación del mundo subjetivo y la del mundo objetivo, al ofrecer una resolución al debate entre esas dos caracterizaciones que tienen primacía en la realidad social (Orlikowski y Robey, 1991). Giddens logra un acercamiento entre lo objetivo y lo subjetivo a través de la crítica al objetivismo de características funcionalista-estructuralista y al subjetivismo que es característico de las sociologías interpretativas y hermenéuticas (fenomenológicas).

Según Giddens (1989), las conductas de los actores individuales reproducen las propiedades estructurales de las colectividades. La Teoría de la Estructuración sostiene que los seres humanos son constructores sociales y sus instituciones son construidas y sostenidas por humanos que actúan de acuerdo a las imágenes que se forman de la realidad. La Teoría de la Estructuración establece que el estudio de la ciencia social está basado en las prácticas sociales, no en la experiencia individual ni en la existencia de cualquier forma de totalidad social.

Las estructuras son prácticas ordenadas recurrentes que no existen al margen de la praxis, de tal forma que la continuidad de estructura necesita del concepto de estructuración, como reproducción de relaciones sociales negociadas a lo largo del tiempo (Giddens, 1981:172).

Por medio del denominado análisis de la conducta estratégica Giddens (1989) toma las instituciones como trasfondo para concentrarse en cómo los actores reproducen las estructuras del sistema en sus prácticas sociales. Giddens no concibe el poder como una simple relación entre individuos, sino como una cualidad estructural de la vida institucional, cronológicamente reproducida por las prácticas comunicativas de sus miembros. A través de las actividades sociales la gente reproduce las acciones que hacen posible esas prácticas sociales que, al institucionalizarse, perpetúan las relaciones de 
poder.

En tal sentido, la tecnología y los sistemas de información (TSI) son concebidos como un conjunto de prácticas sociales que aparecen y evolucionan en el tiempo. La comunicación y transferencia de información, el desarrollo de los sistemas de información y el manejo de conocimiento que involucra las estructuras de la organización, son en esencia el medio y el resultado de la acción humana mediada por las TSI a través del tiempo.

\section{Requerimientos necesarios para analizar un sistema de información en una organización}

Para guiar una investigación acerca de los sistemas de información es útil, en primer lugar hacer el estudio de las intersecciones rutinizadas de las prácticas organizacionales que son los "puntos de transformación" de las relaciones estructurales. En segundo lugar, hay que investigar los modos en que unas prácticas institucionalizadas conectan la integración organizacional con la integración sistémica; así, los sistemas mediados por computadores reafirman las prácticas sociales de las organizaciones.

Los sistemas son conceptualizados como el conjunto de prácticas que, de manera recursiva ejecutan tareas. Al igual que los sistemas sociales, los sistemas de información al estar dedicados a coleccionar, almacenar y diseminar información relevante, pueden ser entendidos como una práctica social de la organización y no como una actividad exclusivamente técnica. Las tecnologías capacitan la recopilación de información que sirve de base para la toma de decisiones. Por ello, las tecnologías y sistemas de información juegan un rol importante en el desarrollo de las actividades que conducen a la interacción de las estructuras (generadoras de rutinas) en los diversos sistemas.

Si consideramos a las capacidades, como aplicaciones que tiene cada organización para realizar actividades que les permita resolver problemas específicos, podemos entonces aseverar que ellas son aplicaciones de conocimiento que sirven para resolver, repetidas veces, los problemas y son, por lo tanto, el resultado de acciones que han funcionado en el pasado. Así que, cuando nos referimos a la organización, implícitamente incorporamos sus experiencias, sus rutinas y la conexión que existe entre ellas, de manera tal que es posible obtener todas las prácticas de la organización, para luego integrarlas en un todo coherente que definimos como el sistema de rutinas. La práctica diaria recurrente corresponde a una rutinización de las reglas, que se expresa incluso en la tecnología utilizada, por lo tanto, la acción mediada por la tecnología implica una conexión inherente entre el agente social y la herramienta. 
Cuando las estructuras se representan en los sistemas y tecnologías se da un proceso de institucionalización que se refuerza con la repetición de las acciones que ejecutan los seres humanos; es por ello que los elementos rutinizados en la práctica diaria pasan a ser parte estructural de la organización. Así, las estructuras se refuerzan en las prácticas organizacionales y se perpet úan con el transcurrir del tiempo.

El desarrollo de sistemas trasciende los aspectos técnicos y por lo tanto posibilita y promueve la participación social dentro de la organización moderna. Como cada sistema puede ser analizado como un conjunto de prácticas sociales rutinizadas, las tecnologías de información las podemos considerar como los recursos materiales que reafirman la práctica informacional que, a su vez, apoyan una práctica social mucho más amplia. Por ello, resulta interesante analizar las estructuras de poder que están implícitas en las decisiones que se toman para desarrollar los sistemas de información.

\section{Sentido de "poder" y las prácticas organizacionales tecnocráticas}

En temas de organizaciones, la búsqueda abierta de poder ha sido considerada como un signo negativo de gerencia. Por ejemplo Kotter (citado por LaPalombara, 2001) encontró que en 2.000 artículos publicados en el Harvard Business Review en un período de veinte años, solamente cinco de ellos incluían la palabra "poder" en sus títulos. Por eso, La Palombara (2001) afirma que el tema del poder es tratado como un secreto sucio de familia: "todo el mundo lo conoce, pero nadie se atreve a discutirlo abiertamente". Nos podríamos imaginar, aunque incorrectamente, que la situación ha cambiado para bien en décadas recientes. Un nuevo examen a la revista Harvard Business Review, con la misma pregunta que se hizo Kotter, muestra que menos de quince de un total de 3.000 artículos publicados en el período comprendido entre 1960 y mediados de 1999 contenían la palabra poder entre sus títulos y que tres de ellos tenían la palabra "conflicto" (La Palombara, 2001).

Las teorías tecnocráticas, según las cuales "el que tiene el conocimiento, tiene el poder" no son nada nuevas. Weber (en Giddens, 2000) señaló al "experto" como una creación de los tiempos modernos, puesto que la racionalidad técnica constituye un factor primordial para el desarrollo del orden productivo. La idea de que "el conocimiento es poder" presenta diversas dificultades puesto que dice poco acerca de quién mantiene el poder (efectivamente) en una situación dada. No siempre, y sin exagerar, tampoco generalmente, aquéllos que poseen conocimientos especializados detentan el poder. Es cierto que existe una posición de "neutralidad" que los tecnócratas tratan de mantener (a 
través de concepciones asumidas y discursos técnicos elaborados) para acercarse al poder, pero ello es debido a que asumir (consciente o inconscientemente) cualquier otra posición podría confrontar a quienes ejercen el verdadero poder en el diseño de políticas que conducen a la toma de decisiones.

Galbraith (1974) afirma que el paso del poder de los propietarios a los directores (tecnocracia) es una realidad que existe y se ejercita cotidianamente; según esa opinión, las complejidades de las organizaciones modernas hacen de la toma de decisiones un hecho que se sustenta en el carácter técnico; por ello, el control tecnocrático, amparado en el conocimiento que los tecnócratas poseen, es la expresión valedera para legitimar la toma de decisiones. Según Galbraith (en Giddens, 2000:201) en la corporación moderna, debido a lo indispensable de la información científica y técnica, aquéllos que poseen el conocimiento especializado de la información, adquieren un poder cada vez mayor dentro de la organización. Sin embargo, no hay razón para suponer que el aumento del control por parte de los gerentes vaya unido a una difusión del poder económico en el seno de una tecno estructura; y es que la consolidación del poder económico no implica que en la jerarquía de la organización, el control del poder se entregue a los técnicos.

La tecnocracia contribuye a la legitimación de las políticas de quienes se apropian del poder, pero su desempeño esencial no es, actuar en una forma objetivamente racional, sino que sirve para propósitos de legitimación de la acumulación del capital, o como lo expresa Ochoa (1995:36) “La tecnocracia debe contribuir a la legitimación, pero su papel esencial es aportar al proceso de acumulación". Para Habermas (en Giddens, 2000) el modo tecnocrático basado en la legitimación técnica de la toma de decisiones políticas, es, de hecho, ideológico. Por lo tanto, debemos estar cautelosos con respecto a la noción de que, en las organizaciones modernas, el poder se ha difundido hacia abajo entre aquellos que poseen experiencia técnica y conocimiento especializado, o que los tecnócratas son quienes deciden en las políticas para la toma de decisiones.

La Teoría de la Estructuración de Giddens nos brinda luces para iluminar ciertos puntos básicos de una teoría del poder, en presencia de una estructura de conocimiento cuando se desarrollan sistemas de información. El poder con significado y trascendencia en las organizaciones consiste en la capacidad para determinar o formular políticas y es en ese contexto, que la formulación de las políticas queda prácticamente en manos de los no especialistas que dirigen la organización. Las decisiones tomadas por la tecno estructura, facilitan o limitan la competencia de las medidas que se toman en instancias de niveles superiores, pero no constituyen una limitación para ponerlas en práctica. De hecho, la tecnocracia no discute las grandes decisiones (las trascendentales) tomadas en el seno de 
la Asamblea de accionista (por ejemplo), antes bien, las apoya y las ejecuta. A la tecnocracia se le relega al papel de ejecución de las políticas que han sido definidas por quienes detentan el poder.

La tecnocracia "controla" que se cumpla, lo que se ha decidido en las altas esferas del poder. Los cargos gerenciales sostenidos por un conocimiento tecnocrático, y no por razones de propiedad, se deben considerar fundamentalmente como una situación relativa a la mediación de los intereses del capital. La creciente dominación de sectores industriales poderosos ha nutrido a las empresas con técnicos (profesionales del área) especializados, que ha devenido en una "revolución de gerentes", tal como lo denomina Giddens (2000). Este auge de "gerentes sin propiedad" sirve para sustentar el funcionamiento de la sociedad tecnocrática, en la que la participación de los gerentes está circunscrita a la mediación del control y no a la mediación institucional del poder.

El carácter ideológico de la tecnocracia reside en servir a los intereses del capital. Los técnicos con conocimiento muy especializado son instrumentos utilizados para acentuar los intereses de los propietarios y su capacitación técnica legitima el modelo de propiedad en la organización. “El modo tecnocrático, basado en la legitimación técnica de la toma de decisiones políticas, es de hecho, ideológico" (Giddens, 2000, p. 306). Para Ochoa (1995), aquellos técnicos que no comparten la ideología capitalista no tienen posibilidades de ascenso a niveles de dirección en las organizaciones tecnocráticas, por lo tanto su poder es limitado a los intereses de los propietarios, es por ello que la tecnocracia es un instrumento de legitimación y de acumulación del capital.

\section{Mercantilización del conocimiento y los sistemas de información a través de alianzas}

A medida que el rol de los sistemas de información ha evolucionado, desde el procesamiento de datos al apoyo de los trabajos basados en el conocimiento, se ha creado la necesidad de integrar los sistemas de las organizaciones, no sólo con los sistemas sociales sino también con los diversos sistemas técnicos que apoyan el uso del conocimiento de la organización. Tal integración supone el desarrollo de nuevas herramientas y estrategias que posibiliten la participación del "conocimiento técnico" en la toma de decisiones. Así, se ha expandido la oferta tecnológica en la resolución de problemas, que involucra a los motores de búsqueda de información en almacenamientos masivos de datos (data-mining y data warehousing), sistemas de apoyo a la toma de decisiones, groupware, herramientas para mapas conceptuales, portales, aplicaciones basadas en tecnología internet (Word Wide Web, servidores web y browsers) aplicaciones 
de intranet (uso de tecnología internet con acceso restringido a miembros de las organizaciones participantes), todos ellos fácilmente mercantilizados en el proceso de globalización.

Así mismo, las decisiones gerenciales, con relativa frecuencia, están tentadas en adoptar soluciones radicales a problemas organizacionales. Por ejemplo, empresas de gran tamaño han utilizado en forma drástica técnicas de externalización (tercerización u outsourcing) para resolver conflictos tanto técnicos (tecnología, costos, innovación, etc.) como sociales (adiestramiento, aprendizaje, laboral, etc.). Estas prácticas organizacionales hacen énfasis en la "externalización" de los conflictos funcionales; ello implica suponer que el conocimiento puede ser tratado como un bien o como un activo de las organizaciones que se puede mover, trasladar y mercadear.

Muchas empresas trasladan sus operaciones rutinarias a organizaciones especializadas, reservando la toma de decisiones para aquellas situaciones que son vitales para sus asuntos. Así, el poder de decisión es diversificado con el uso de las TI y con la especialización del conocimiento técnico. En el pasado, las organizaciones realizaban sus propias actividades de procesamiento de datos; sin embargo, con el correr del tiempo esos servicios se han venido especializando cada vez más, de forma tal que con mucha frecuencia, las empresas traspasan las operaciones relacionadas con TSI a otras empresas especializadas, y desarrollan, de esta manera, relaciones gerenciales a través de diversas tipos de alianzas, particularmente las denominadas outsourcing de los sistemas de información.

El outsourcing de los servicios de computación se inició a principios de los años 60, cuando las compañías comenzaron a contratar con terceros las tareas relacionadas con el procesamiento de datos. Las empresas veían como la velocidad de crecimiento del procesamiento de datos en sus propios sitios de trabajo aumentaba, al igual que lo hacían los costos en tecnologías de información que se requerían para apoyar las áreas de finanzas y operativas, cuando los computadores eran muy costosos y físicamente muy grandes al punto que requerían mucho espacio para su instalación; así, los costos no estaban asociados directamente a la producción o a las actividades vitales de las organizaciones. En la década de los sesenta, el principal costo asociado a los TSI era el del hardware, mientras que en la década de los setenta el gasto por desarrollar software era la pricnicpal preocupación. Por lo tanto, el outsourcing en el área de los TSI no es nada nuevo, su aparición es evidente en la era del procesamiento de datos a través del uso de programadores y la compra de paquetes de software (Currie, 1995). La década de los noventa se caracterizó por un interés renovado por las políticas de outsourcing, pero con 
nuevas formas de implementación. Mientras en los sesenta, los servicios asociados al procesamiento de datos se realizaban afuera de la organización contratante y la empresa que ofrecía el servicio utilizaba sus propios equipos, la política de outsourcing desarrollada a partir de los noventa, utiliza las instalaciones de la organización contratante, incluso contratan, absorben o trasladan el recurso humano existente y utilizan los equipos de la propia organización contratante.

Las funciones de los sistemas de información se han convertido en un objetivo primordial para el desarrollo del outsourcing como técnica empresarial, y lo demuestra el que cada vez más empresas a nivel mundial buscan alianzas de este tipo para desarrollar sus sistemas. Hoy en día, el outsourcing de TSI es muy popular en las empresas de comunicaciones, computadores y semiconductores. Según la revista Fortune 500 (en Malhotra, 1995) a principio de los noventa el $42 \%$ de empresas de comunicaciones, $40 \%$ de los fabricantes de computadores y $37 \%$ de las compañías de semiconductores confiaron en la política de outsourcing con empresas extranjeras para desarrollar productos.

Cada vez con más frecuencia, las empresas grandes ponen afuera el "control" total de sus procesos y funciones de sistemas de información, y ello ha sido así, a través del "outsourcing de los sistemas de información". Por ejemplo, en 1989 la empresa Enrol Corporation inició, en todo el mundo, la primera relación significativa de outsourcing en sistemas de información, con un contrato que alcanzó la suma de 750 millones de dólares, secundado por Kodak en 1990, con un contrato diversificado en tres empresas de sistemas de información (Field, en Yost y Harmon, 2002). Desde entonces, este tipo de alianzas ha experimentado un crecimiento de tal magnitud que en los Estados Unidos pasó de los 9 billones de dólares en 1990 a 40 billones en 1998 (Yost y Harmon, 2002). Las empresas dedicadas al área de desarrollo de productos de computación (software, sistemas y hardware) han crecido rápidamente apoyadas por estrategias de outsourcing. Empresas como Dell Computer creció a una rata del $89 \%$ por año en forma sostenida durante la década de los ochenta, con un ritmo de ventas del orden de los $\$ 700.000$ (dólares) por empleado. La gerencia del conocimiento fue la estrategia principal que desarrolló Dell para poder atender a sus clientes en forma directa. Dell desarrolló una estrategia de outsourcing para atender a clientes grandes, basada en el conocimiento que se consolidaba en períodos largos, en lugar de dedicarse a una estrategia de corto plazo diseñada para disminuir costos (Quinn, 1999).

Para este tipo de alianza, por lo general, se parte de la idea de que los sistemas y tecnologías de la información existen única y exclusivamente para sustentar y apoyar los 
procesos productivos de la organización. En tal sentido, si la organización no tiene dentro de sus objetivos de producción, el manejo de datos y tratamiento de la información entonces esas actividades no se consideran vitales para el desarrollo de la organización. Ante esa situación, la gestión de las infraestructuras tecnológicas no justifican la contratación de profesionales especializados en esas áreas, y por lo tanto, se concentran en otras actividades y tareas de mayor valor para la organización, especialmente cuando se puede delegar en un suministrador externo que se encuentre capacitado para asumir la responsabilidad del manejo de información en forma total o parcial.

El outsourcing es una estrategia gerencial que transfiere afuera de la organización las actividades no vitales, para que otros entes especializados las ejecuten en forma más confiable, más eficiente y más económica. No es un contrato a corto plazo, ni se limita a una asesoría puntual en un área de especialidad determinada; tampoco consiste en la contratación de personal experto por un breve lapso para mejorar puntos específicos del servicio. Se trata, más bien, de la entrega de un conjunto de necesidades y requerimientos relacionados con actividades que no son el objetivo de la organización, para que un agente externo las desarrolle.

El gran dilema que pareciera enfrentar una organización para decidir si desarrolla los sistemas de información "en casa" o los entrega para ser desarrollados "afuera" está en función de los costos. ¿Costará más construir los sistemas de información en casa, que establecer una relación de outsourcing?. ¿Costarán más los actividades propias de la empresa con sistemas de información desarrollados internamente, que si estuvieran desarrollados en outsourcing?

Además de la reducción de costos, otras muy importantes razones existen para llevar a cabo el outsourcing y tienen que ver con el acceso a habilidades especializadas, reducción del reclutamiento y entrenamiento, economías de escala, altos controles de estándares de calidad, proliferación de los diversos servicios informacionales y seguridad. Es por ello que, de los factores determinantes para realizar outsourcing destacan las consideraciones financieras y las estrategias de los negocios; pero indudablemente, desde un punto de vista técnico, las funciones de los SI en relación con la productividad es el factor más importante porque provee una perspectiva más “objetiva” para su concreción.

Aún, a pesar de la diversidad de razones que puedan existir para que una organización se sienta atraída para desarrollar alianzas estratégicas en los servicios de sistemas de información, siempre existe una tendencia a mantener en "casa" el desarrollo de algunas aplicaciones de software. Las razones varían de acuerdo al tipo de intereses que pudieran 
las organizaciones tener y que se relacionan con la necesidad de acomodar sus servicios a particularidades de la organización, confidencialidad de los datos e información, el deseo por reducir los riesgos asociados a los agentes externos, facilidad para desarrollar aplicaciones y adaptar software, y la necesidad por desarrollar competencias en tecnologías y sistemas de información.

Una de las principales críticas que se pueden argumentar al desarrollo de la política de outsourcing se refiere a la pérdida del control sobre los activos de TSI, tales como: amenaza de los proveedores de aprovecharse de las vulnerabilidades en la entrega de activos; pérdida de la experticia en materia de SI, pérdida de la memoria colectiva; posibilidad de declinación de la moral y el rendimiento de los empleados, y, quizás los más controversial, el desbalance que se presenta entre las implicaciones estratégicas y la rentabilidad financiera.

\section{Políticas de outsourcing de los SI en las empresas del Estado}

Las organizaciones públicas del Estado también desarrollan alianzas de tipo outsourcing en las operaciones asociadas a las tecnologías y sistemas de información (TSI). Existen en el mundo ejemplos puntuales donde el sector público ha utilizado el outsourcing para las actividades de TSI. Tales prácticas organizacionales se han aplicado en áreas vitales como son: el impuesto sobre la renta y el sistema de distribución de la beneficencia pública (welfare) en Inglaterra; el sistema de control de tráfico aéreo de Nueva Zelanda; el sistema de recolección de pagos por violaciones de tráfico automotriz en la ciudad de Chicago; la automatización del sistema de cruce de las fronteras en Singapore (Peled, 2000). En Venezuela, la más importante empresa del país (PDVSA) desarrolló, a partir de 1997 una política de outsourcing en el área informática y de telecomunicaciones con una empresa extranjera, Science Applications International Corporation (SAIC), cuyo negocio era el desarrollo de tecnologías y sistemas de información, a través de la constitución de una empresa de servicios informáticos que se llamó Informática, Negocios y Tecnología, S.A. (INTESA).

Con el outsourcing de empresas del Estado, se transfieren los recursos organizacionales (inmuebles y empleados) a un tercero; en retribución, el tercero provee al Estado de una garantía amplia en el desarrollo de los servicios acordados y en el logro de ciertos niveles de servicio. En el área de los sistemas y tecnologías de información, con el outsourcing, por lo general, se incluyen la gerencia y operación de las instalaciones de computación, el mantenimiento de las redes de información, el desarrollo de infraestructura y aplicaciones de computación y, el entrenamiento y apoyo a los usuarios. 
El estudio del outsourcing de las $\mathrm{TI}$ en empresas del Estado no ha sido tratado con profundidad por investigadores y escuelas académicas. Por ello, existen pocas fuentes de información que ayuden a explorar y analizar alternativas en la definición del outsourcing de las tecnologías de información en dichas empresas. La poca literatura que existe en relación con el tema del outsourcing ignora su dimensión política, puesto que presta más atención a la producción, costos de transacción, empleo, reestructuración e impacto entre otros, sin 'restarle mucha importancia a los aspectos menos tangibles y menos cuantificables, pero que son determinantes a la hora de llevar a cabo una alianza. Los estudios publicados que evalúan las características menos tangibles del outsourcing por lo general destacan los aspectos "suaves" de las tecnologías de información, como son la socialización de los especialistas y técnicos cuando proveen servicios y las ventajas en la atención de usuarios finales. Una importante interpretación que se tiene del porqué se tratan en la administración pública los aspectos asociados a las dimensiones técnicas y financieras del outsourcing es debido a que este tipo de estrategia y práctica gerencial es manejada como si se tratara de una empresa privada. Así que, en la administración pública, las relaciones del outsourcing con la política, poder, influencia y control gerencial no han sido consideradas con profundidad y por lo tanto, no ofrecen luces acerca de cómo los miembros de la burocracia pública y los vendedores del servicio manipulan su conocimiento tecnológico y político para controlar los proyectos de tecnologías y sistema de información.

Una apreciación muy interesante acerca de la definición de outsourcing es la expresada por Gordon y Walsh (1997:267) quienes afirman que el outsourcing es “... una forma distinta de privatización donde las funciones particulares de las instituciones públicas son transferidas al sector privado". Willcocks y Currie (1997:35) sugieren que el outsourcing de TSI en el sector público ha sido manejado por dos iniciativas particulares: por un lado, la reducción de costos y, por el otro, una expandida creencia de que las empresas del sector privado son más eficientes. Adicionalmente, se tiene la creencia de que en el sector público se carece de habilidades en TSI, poca posibilidad de acceso a los conocimientos recientes en el área de las TSI, una desproporción en el sistema de pago del sector público en relación con sus pares del sector privado, una creencia generalizada de los gerentes del sector público que en el sector privado se desarrollan mejores trabajos y en tiempo oportuno, así como la falta de confianza que se tiene por la administración pública general.

Nuestra definición de outsourcing en la administración pública se expresa de la siguiente manera: El outsourcing en las empresas públicas debe ser entendido como la evolución de 
las complejidades tecnológicas y de las relaciones políticas que ocurren entre las empresas públicas que se someten a la alianza (la burocracia) y el tercero quien ofrece los servicios (vendedor privado) después de que los dos se han unido formalmente para producir nuevos productos y servicios asociados a las tecnologías y sistemas de información.

Con esa definición de outsourcing, reconocemos que los terceros asumen un rol de liderazgo cooptando con la burocracia pública en el financiamiento y el diseño tecnológico, cuyo resultado es un tipo de organización que se apropia de una relación políticotecnológica. Así, la burocracia puede jugar un rol crítico en materia tecnológica al influenciar en las esferas apropiadas, apoyando las decisiones técnicas del proyecto. La burocracia pública y los agentes terciarios cooperan directamente entre ellos en las dimensiones política, organizacional y tecnológica del proyecto y en forma indirecta en la dimensión financiera. Y ello es así, porque la burocracia pública tiene amplia fortaleza en la administración de los recursos del Estado, por lo general con experiencia gerencial (no necesariamente en los aspectos públicos), que les permite ascender en la escalera burocrática al mostrar lealtad a los valores éticos construidos en la organización y que son parte del código que está expresado en la misión y visión que se les ha inculcado previamente, los cuales están asociados al rol que ejercen en la organización, así, tal como los expresa Stever (en Peled, 2000), la burocracia se ve como guardiana de los más amplios intereses públicos y está sujeta a las más severas críticas, si ella no se comporta adecuadamente. Por la otra parte, los terceros (vendedores del outsourcing en $\mathrm{TI}$ ) poseen un entrenamiento tecnológico extensivo en materia de TSI, poseen experiencia y están incentivados para adquirir nuevas habilidades técnicas. No tienen lealtad con la cuestión pública, de hecho con bastante frecuencia la menosprecian, puesto que no comparten valores con la burocracia. Sólo están a su lado por la motivación que les da el desarrollo del proyecto tecnológico que los vincula, por lo tanto su acompañamiento es por un período corto, lo que dure la alianza y en las condiciones definidas por las obligaciones contractuales. Son, en sus compromisos organizacionales, parte de una nueva clase técnica nómada que se mueve en forma rápida entre las asignaciones de nuevos contratos de outsourcing que resultan muy lucrativos. Estos vendedores buscan servir a los segmentos más enriquecidos de las organizaciones y por lo tanto, prestan poca atención al compromiso y desarrollo social de sus acciones.

Desde un punto de vista estrictamente técnico, las organizaciones públicas se someten al outsourcing, básicamente porque ellas no pueden manejar la complejidad tecnológica de los sistemas de información, y ello es debido a las crecientes dificultades que experimentan al reclutar y mantener un personal técnicamente calificado y, por la 
frecuencia con que se falla en el reparto en forma oportuna de productos de TI que sean eficientes y que se adecuen al presupuesto. Los servidores públicos que son expertos en TI les cuesta mucho ser eficientes en la construcción de sistemas de información, ello es debido a que el número de componentes tecnológicos en un sistema (interfases, PCs, servidores, protocolos de comunicación, redes, líneas telefónicas y modelos de software) son tan complejos y variados que se vuelven poco manejables, así que la probabilidad de falla en los sistemas de computación antes de ser repartidos es muy alta, debido, entre otras cosas, a que no pueden ser todos probados con anterioridad. También, se les hace muy difícil recopilar necesidades y escribir buenos requerimientos para proyectos de tecnologías y sistemas de información, a la vez que les cuesta separar la planificación de la ejecución, sobre todo en organizaciones donde los intereses técnicos y profesionales están disociados entre los participantes.

Adicionalmente, las complejidades de los sistemas de información en el sector público son muy altas, principalmente por las propias complejidades de la legislación que subyacen en los procesos organizacionales del sector público. Requerimientos de proyectos adecuados demandan conocimiento de la organización y de los aspectos técnicos; sin embargo, los servidores públicos no pueden mantener el ritmo de cambio tecnológico y los terceros raras veces conocen a profundidad la organización para identificar tales requerimientos; por ello, con frecuencia, los vendedores aplican las soluciones genéricas que han desarrollado con anterioridad en otras organizaciones (públicas o privadas), en lugar de diseñar soluciones desde cero para una en particular.

La estrategia de outsourcing de TSI en el sector público ha devenido en experiencias no satisfactorias, debido, principalmente, al número de disoluciones que genera, a la inestabilidad de sus procesos operacionales y a la pérdida de control de operaciones no vitales pero que son estratégicas para el desarrollo de las actividades. Así, la toma de decisiones es favorecida por quienes detentan los intereses que se expresan en el poder que ejerce la clase tecnocrática, la cual se instala en los niveles de la burocracia pública por un período que no los compromete con los intereses públicos, desarrollándose entre el sector privado y el sector público prácticas organizacionales que sirven para intensificar las relaciones de poder presentes en la organización.

\section{Reflexiones finales}

Esta investigación contribuye a entender el desarrollo de los sistemas de información en las organizaciones, porque se centra en los aspectos asociados al poder. Nuestro estudio investigó las prácticas rutinarias que llevan a cabo los miembros de las organizaciones, en 
particular la de los especialistas y técnicos y su vinculación con las relaciones de poder en un sistema de propiedad que le es ajeno. De tal forma, que la principal contribución de esta investigación consiste en clarificar que los sistemas de información pueden ser analizados en términos de una conceptualización social que permite comprender en profundidad cómo las personas con conocimiento técnico utilizan las prácticas organizacionales para acentuar sus intereses y afianzar su poder.

El desarrollo de los SI utiliza no sólo las herramientas técnicas que son requeridas, sino también las relaciones que acentúan las estructuras organizacionales que consolidan el poder. Por ello, la investigación se centró en la noción de rutinización como práctica de institucionalización organizacional objetivada en las tareas y actividades expresadas en el ejercicio del poder.

Esta investigación realiza aportes a la comprensión de los conflictos presentes en las organizaciones que desarrollan políticas de alianzas en el uso de TI, en particular, en el estudio de las políticas de outsourcing de los SI, puesto que existe una carencia de investigaciones de las prácticas asumidas en el proceso de entregar a terceros las decisiones que en materia de desarrollo de SI realizan las organizaciones, y mucho más escasean investigaciones si se trata de empresas públicas.

Los estudios tradicionales de outsourcing muy pocas veces tratan las alianzas en materia de sistemas y tecnologías de información, y los pocos que existen, lo hacen sobre la base del estudio de contratos con base a términos económicos en donde la alianza es entendida y analizada con el lente de las relaciones costos/beneficios y de corto plazo, en lugar de hacerlo con la apreciación de los términos poder, conocimiento y legitimación de las estructuras a través de la práctica organizacional. Por ello, este estudio hace una contribución distinta e importante al análisis de los sistemas de gestión de información.

La interpretación que hicimos del ejercicio del poder demandó un esfuerzo extra al que se hubiese necesitado si se limitara a un análisis económico o financiero, puesto que investigar y comprender el desarrollo del outsourcing en empresas del Estado, requiere de una forma alternativa de análisis a los estudios que se realizan en empresas privadas. Así que el propósito principal del uso de la Teoría de la Estructuración en el estudio de los sistemas de información es contribuir al análisis crítico del desarrollo social y organizacional de los sistemas, a través de la exposición de las consecuencias sociales que se esconden en las políticas de outsourcig.

Con esta investigación se ha comenzado a explorar las implicaciones del análisis del 
outsourcing que podrían ser útiles para desarrollar un marco conceptual de corte crítico sociológico que permita entender mejor, cómo las propiedades técnicas de los sistemas de información pueden facilitar y colaborar en la construcción de una nueva gestión organizacional.

\section{Referencias Bibliográfica}

1. Currie, Wendy (1995), Outsourcing the new IT strategy. En Currie, W. (Eds.), Management for IT: An I nternational Perspective, Pitman Publishing, London.

2. Galbraith, John (1974), El Nuevo Tratado Industrial. Editorial Aries. Sexta edición. Barcelona, España.

3. Giddens, Anthony (1981), Agency, Institution, and time-space analysis. En KnorrCetina y Cicourel (Eds), Advances in Social Theory and Methodology. Boston MA Routledge \& Kegan.

4. Giddens, Anthony (1989), The constitution of society: Outline of a Theory of Structuration. Berkerly, CA. University of California Press.

5. Giddens, Anthony (2000), La estructura de clases en las sociedades avanzadas. Alianza Universidad. Editorial Alianza. España.

6. Gordon, Mark y Walsh, Timothy (1997), Outsourcing technology in government: owned, controlled, or regulated institutions. Journal of Government Information, Volúmen 24, Número 4, pp 267-283.

7. LaPalombara, Joseph (2001), Power and Politics in Organizations Public and Private Sector Comparisons. En Meinolf Dierkes (Eds.) et al, Handbook of Organizational Learning and Knowledge. Oxford University Press.

8. Malavé, José (1999), Prácticas Organizacionales. Una perspectiva de procesos en la teoría de la organización. Caracas. IESA.

9. Malhotra, Yogesh (1995), An Empirical Analysis of the Determinants of Information Systems Productivity and the Role of Outsourcing Policy. Recuperado de http://www. brint. com/papers/outsourc/. Más reciente visita: 02 de febrero de 2004 
10. Ochoa, Haydée (1995), Tecnocracia y Empresas Públicas en Venezuela (1948 1991). Universidad del Zulia. Vicerrectorado Académico. Maracaibo, Venezuela.

11. Orlikowski, Wanda y Robey, Daniel (1991), Information Technology and the Structuring of Organizations. Information Systems Research,. The Institute of Management Sciences. Volúmen 2, Número 2, pp143-169.

12. Peled, Alon (2000), The politics of outsourcing: bureaucrats, vendors, and public information technology (IT) projects. Information I nfrastructure and Policy, Número 6, pp 209-225.

13. Quinn, James (1999), Strategic outsourcing: leveraging knowledge capabilities. Sloan Management Review, Número Summer.

14. Willcocks, Leslie y Currie, Wendy. (1997), Contracting-out information technology in the public sector context: research and critique. Journal of the Australian and New Zeland Academy of Management, Volúmen 2 Número. 2, pp 34-49.

15. Yost, Jeffrey y Harmon, Ken (2002), Contracting for Information Systems Outsourcing with Multiple Bidders. Journal of Information Systems, Volumen 16, Número 1, Spring, pp 49-59. 